\title{
Motor and Predictive Processes in Auditory Beat and Rhythm Perception
}

\author{
Shannon Proksch, Daniel C. Comstock, Butovens Médé, Alexandria Pabst and \\ Ramesh Balasubramaniam*
}

Sensorimotor Neuroscience Laboratory, Cognitive \& Information Sciences, University of California, Merced, Merced, CA, United States

In this article, we review recent advances in research on rhythm and musical beat perception, focusing on the role of predictive processes in auditory motor interactions. We suggest that experimental evidence of the motor system's role in beat perception, including in passive listening, may be explained by the generation and maintenance of internal predictive models, concordant with the Active Inference framework of sensory processing. We highlight two complementary hypotheses for the neural underpinnings of rhythm perception: The Action Simulation for Auditory Prediction hypothesis (Patel and Iversen, 2014) and the Gradual Audiomotor Evolution hypothesis (Merchant and Honing, 2014) and review recent experimental progress supporting each

Edited by: Marta Olivetti Belardinelli, Sapienza University of Rome, Italy

Reviewed by:

Bruno Laeng,

University of Oslo, Norway Riccardo Brunetti,

European University of Rome, Italy

${ }^{*}$ Correspondence: Ramesh Balasubramaniam ramesh@ucmerced.edu

Specialty section:

This article was submitted to Cognitive Neuroscience, a section of the journal Frontiers in Human Neuroscience

Received: 30 June 2020 Accepted: 18 August 2020 Published: 11 September 2020

Citation:

Proksch S, Comstock DC,

Médé B, Pabst $A$ and Balasubramaniam R (2020) Motor and Predictive Processes in Auditory

Beat and Rhythm Perception. Front. Hum. Neurosci. 14:578546. doi: 10.3389/fnhum.2020.578546 of these hypotheses. While initial formulations of ASAP and GAE explain different aspects of beat-based timing-the involvement of motor structures in the absence of movement, and physical entrainment to an auditory beat respectively-we suggest that work under both hypotheses provide converging evidence toward understanding the predictive role of the motor system in the perception of rhythm, and the specific neural mechanisms involved. We discuss future experimental work necessary to further evaluate the causal neural mechanisms underlying beat and rhythm perception.

Keywords: beat perception, motor system, motor planning, sensorimotor system, rhythm, timing

\section{INTRODUCTION}

The coupling of action and prediction in perception has been characterized by predictive models of perception (Rao and Ballard, 1999) including classical Predictive Coding (Friston, 2002, 2005) (PC), and the more recent Active Inference Framework (Active Inference - corollary to the Free Energy Principle, Friston et al., 2009; Friston, 2010; Parr and Friston, 2019). Under classical PC, the brain is thought to utilize an internal generative model and a process of probabilistic model updating to predict the causes of its sensory input. Each level of the neural hierarchy predicts the activity at the level below, with higher levels of the hierarchy providing empirical priors, or hypotheses that constrain the generation of new priors at the level below. At each level, the topdown predictive signal is compared to the bottom-up inputs from the lower level. When there is a mismatch between incoming, bottom-up sensory information and top-down predictions, a prediction error is propagated back to the level above where it is used to revise and improve the initial hypothesis. If the prediction error cannot be minimized at the level at which it is being 
processed, it is relayed up to the next level above. The higher in the hierarchy the prediction error is being relayed, the more substantial the revision in the hypothesis. Perceptual experience arises as prediction error is minimized and a 'winning' hypothesis is selected. Thus, the general idea of PC is perceptual inference.

However, this classical PC/Bayesian account of perception characterizes the brain as a passive, Helmholtzian, stimulusresponse machine, responsive only to the generation of prediction errors between its top-down sensory predictions and the actual sensory input from the world (Friston and Stephan, 2007; Clark, 2013). Our brains are more aptly described as embodied and enactive, enabling us to move and interact with our environment to bring about the minimization of prediction errors through our own action (Thompson, 2007; Gallagher et al., 2013; Bruineberg et al., 2018). This is the premise of Active Inference. As in PC, the brain uses an internal generative model to predict incoming sensory data. However, rather than relying on the passive accumulation of bottom-up sensory prediction errors that are minimized to create the content of perception, Active Inference formulations incorporate active engagement with the world to make the sensory inputs more predictable. Thus, in Active Inference, the prediction error minimization process which gives rise to perceptual experience is achieved through actions which conform sensory inputs to the brain's predictions (Friston et al., 2009; Hohwy, 2013; Parr and Friston, 2019).

Music perception and production are exemplar cognitive and behavioral phenomena to study these predictive processes and to evaluate the role of motor processing in sensory perception. Koelsch et al. (2019) expanded on the specific properties of music which make it an ideal paradigm for investigating predictive processes in the brain. Music, in any culture, is based on the generation of regularities, from the temporal regularities of rhythm to the predictable patterns and combinations of musical pitches. These regularities, or expectancies, generated by music have even been proposed as the properties which underlie emotional experience in music (Meyer, 1956; Huron, 2008; Juslin and Västfjäll, 2008). Cross-cultural perceptual priors may exist for some aspects of rhythm perception and production (Jacoby and McDermott, 2017), while other aspects are shaped by encluturation within a certain musical niche (Cameron et al., 2015; van der Weij et al., 2017; Polak et al., 2018). In particular, the experience of musical groove, that property of 'wanting to move' to the music, is proposed to be related to the balance between prediction and prediction errors generated by rhythmic properties of the music (Janata et al., 2012; Matthews et al., 2019, 2020). Active Inference formulations account for not only predictions related to expected stimulus input, but also predictions related to the expected accuracythe precision, or uncertainty-of the original sensory prediction, in addition to counterfactual predictions related to how these prediction errors and their precision would change in response to active motor engagement with the sensory stimulus. Expected precision is modulated by sensory context and active engagement with the sensory signal. The generation of internal, predictive sensorimotor timing signals aligned to the musical beat may enhance the prediction and precision of temporal expectancies when perceiving syncopated musical rhythms, such as in musical groove (Koelsch et al., 2019). Whether or not we actually move our bodies to a musical rhythm, interactions between sensory and motor systems in our brain have been theorized to generate predictive timing signals that help us process musical rhythm (Merchant and Honing, 2014; Patel and Iversen, 2014; Vuust and Witek, 2014). These predictive timing signals are what allow for beat induction, or the active detection of the pulse in rhythmic time-varying stimuli such as music (Honing et al., 2014).

While predictive theories or perception are not new [indeed, they precede the age of Helmholtz, dating as far back as the 11th century works of Arab scholar al-Haytham et al. (ca. 1030; 1989)], the purpose of this review is to contextualize recent advances in the role of the motor system in rhythm and musical beat perception under more recent advances within the Active Inference framework. We then directly compare two hypotheses for the neural underpinnings of rhythm perception: The Action Simulation for Auditory Prediction (ASAP) hypothesis (Patel and Iversen, 2014) and the Gradual Audiomotor Evolution (GAE) hypothesis (Merchant and Honing, 2014). We suggest that the both hypotheses-taken together under the umbrella of Active Inference-provide converging evidence toward understanding the predictive role of the motor system within a distributed sensorimotor network underlying the perception of rhythm.

\section{ACTION AND PREDICTION IN RHYTHM PERCEPTION}

The role of the motor system in rhythm perception is most obviously recognized by examining how it is we engage our body with music. In addition to beat induction in passive music listening, humans - and a limited group of birds and mammals (Kotz et al., 2018; Ravignani et al., 2019) - can move in time to a musical beat. This process of rhythmic entrainment is defined as the ability to flexibly perceive and synchronize to the beat of music or other complex auditory rhythms. It is argued that rhythmic entrainment abilities are determined by the ability to perceive a beat, the underlying pulse, within rhythmic stimuli. Beat perception in humans is inherently predictive, constructive, hierarchical, and modality biased. In addition, beat perception engages the motor system, even when no movement is present (Grahn and Brett, 2007; Chen et al., 2008a,b; Gordon et al., 2018).

In humans, behavioral evidence for prediction in beat perception comes from tapping experiments that reveal negative mean asynchronies, which are not observed in other primates. Asynchronies are observed when humans tap slightly earlier or later than the beat in a rhythmic stimulus, and negative mean asynchronies are a behavioral indicator that humans actively anticipate upcoming stimuli. Mean tapping asynchronies throughout a rhythmic stimulus are usually negative in the auditory domain, but much more variable in the visual domain (Pabst and Balasubramaniam, 2018). Humans also adjust future tapping response based on temporal mismatch between their movement and the current beat (Balasubramaniam et al., 2004), and overtly tapping along to the beat aids in forming temporal predictions when compared to passively tracking a beat (Morillon and Baillet, 2017). In addition, when visual stimuli are presented 
in a way that indicates movement over time, e.g., apparent hand motion (Hove and Keller, 2010) or a bouncing ball (Iversen et al., 2015), predictive entrainment as demonstrated by negative mean asynchrony becomes much more successful.

According to Active Inference, the brain minimizes prediction error either by updating predictions or by taking action in the world to bring actual proprioceptive input in line with top-down predictions regarding driving sensory stimuli. In musical beat perception, this means that we either take action and move to the beat, or we update our predictions by suppressing actual movement and instead establishing an internal model of the beat which corresponds to the proprioceptive input we would have received had we actually been moving to the beat. The ability to flexibly adapt motor behavior in response to a mismatch between a rhythmic auditory stimulus and current motor movement (Balasubramaniam et al., 2004) can be construed as one example of this more general active inference process. Enhanced rhythmic entrainment abilities for the visual domain when visual stimuli implies movement (Hove and Keller, 2010; Iversen et al., 2015), and the improvement of temporal predictions in conjunction with overt rhythmic movement (Morillon and Baillet, 2017) can also be explained by the increase of sensory information available in order to update and modulate descending predictions about the temporal regularities of the stimulus which guide motor movements.

But this Active Inference gloss on beat perception is by itself - vague. Plausible neural architectures have been proposed to support the classical (Helmholtzian) PC/Bayesian processing of music in general (Friston and Friston, 2013). However, an empirically detailed account of the specific neural underpinnings of embodied Active Inference in human musical beat perception is necessary. The motor system has been proposed to play a key role in prediction and perception of sensory information (Schubotz, 2007), and is functionally organized to enable the driving (ascending) and modulatory (descending) message passing hypothesized within the Active Inference Framework (Adams et al., 2013). This differs slightly from traditional theories of motor control, where driving signals arise from descending, top-down motor commands. Under Active Inference, top-down predictive signals from the motor system serve to modulate proprioceptive predictions regarding driving, feed-forward sensory signals (Adams et al., 2013).

Concordantly, the motor system has been found to be consistently active when listening to music, even in the absence of specific motor movement. A recent meta-analysis of fMRI studies found clusters of activations in key regions of the motor system in passive music listening, including bilateral premotor cortex and right primary motor cortex (Gordon et al., 2018). Metrical musical stimuli have also elicited activation in the basal ganglia, supplementary motor area, and cerebellum (Grahn and Rowe, 2009). Indeed, the modality bias for human beat perception and rhythmic entrainment for auditory stimuli (Pabst and Balasubramaniam, 2018), and improvements of auditory beat processing when making overt action (Morillon and Baillet, 2017) can be explained by tight connections between auditory and motor regions of the brain. But the activation of motor structures of the brain, even in the absence of overt movement, indicates that the motor system plays a more fundamental role in the formation of abstract predictive models which support sensory perception (Schubotz, 2007; Adams et al., 2013; Patel and Iversen, 2014).

Strong explanations of rhythm perception must account not only for prediction in action, but also for the role of the motor activity observed in passive music listening. Below, we provide an overview on the motor system's role in rhythm perception, and review two complementary hypotheses which highlight the causal role of the motor system in beat-based timing perception.

\section{MOTOR SYSTEM IN RHYTHM PERCEPTION: VIEWS FROM THE ACTION SIMULATION FOR AUDITORY PREDICTION AND THE GRADUAL AUDIOMOTOR EVOLUTION HYPOTHESES}

Rhythm perception involves two types of timing perception, interval-based (absolute) timing and beat-based (relative) timing (Grube et al., 2010; Ross et al., 2016a; Iversen and Balasubramaniam, 2016). Interval-based timing refers to the ability to discriminate absolute differences in interval duration, whereas beat-based timing refers to the ability to measure the duration of time intervals relative to underlying temporal regularities such as beats (Teki et al., 2011). Beat-based timing perception is thought to be uniquely human (Merchant and Honing, 2014), and is believed to rely on the formation and maintenance of internal predictive models. According to the ASAP hypothesis (Patel and Iversen, 2014), these internal predictive models consist of periodic motor planning activity communicated via the dorsal auditory stream which allow for auditory prediction in beat-based musical timing perception. ASAP highlights the dorsal auditory stream due to its structural and functional relationship between auditory and motor planning regions, facilitating temporally-precise two-way signaling between these regions. This neural pathway involved in spatial processing of sounds (Rauschecker and Tian, 2000; Patel and Iversen, 2014) is more developed in humans than nonhuman primates, which is consistent with differences in beatbased timing behavioral ability (Honing, 2012; Patel and Iversen, 2014). In addition, Rauschecker (2018) postulates that the dorsal auditory stream may also be forming an "internal model of the outside world... [which] conver[ts] sensorimotor sequences into a unified experience"(p264-5). In the case of musical beat-based timing perception, the dorsal stream should form an internal model of the periodic musical beat.

Complementary to the ASAP hypothesis, the GAE hypothesis has been proposed to account for differences in beat-based temporal processing between primates and humans (see Figure 1 for an overview comparison of ASAP and GAE). The GAE hypothesis (Merchant and Honing, 2014) also posits the dorsal auditory stream as a potential substrate for rhythm entrainment and perception. However, GAE claims that the evolution of rhythmic entrainment results more specifically from 


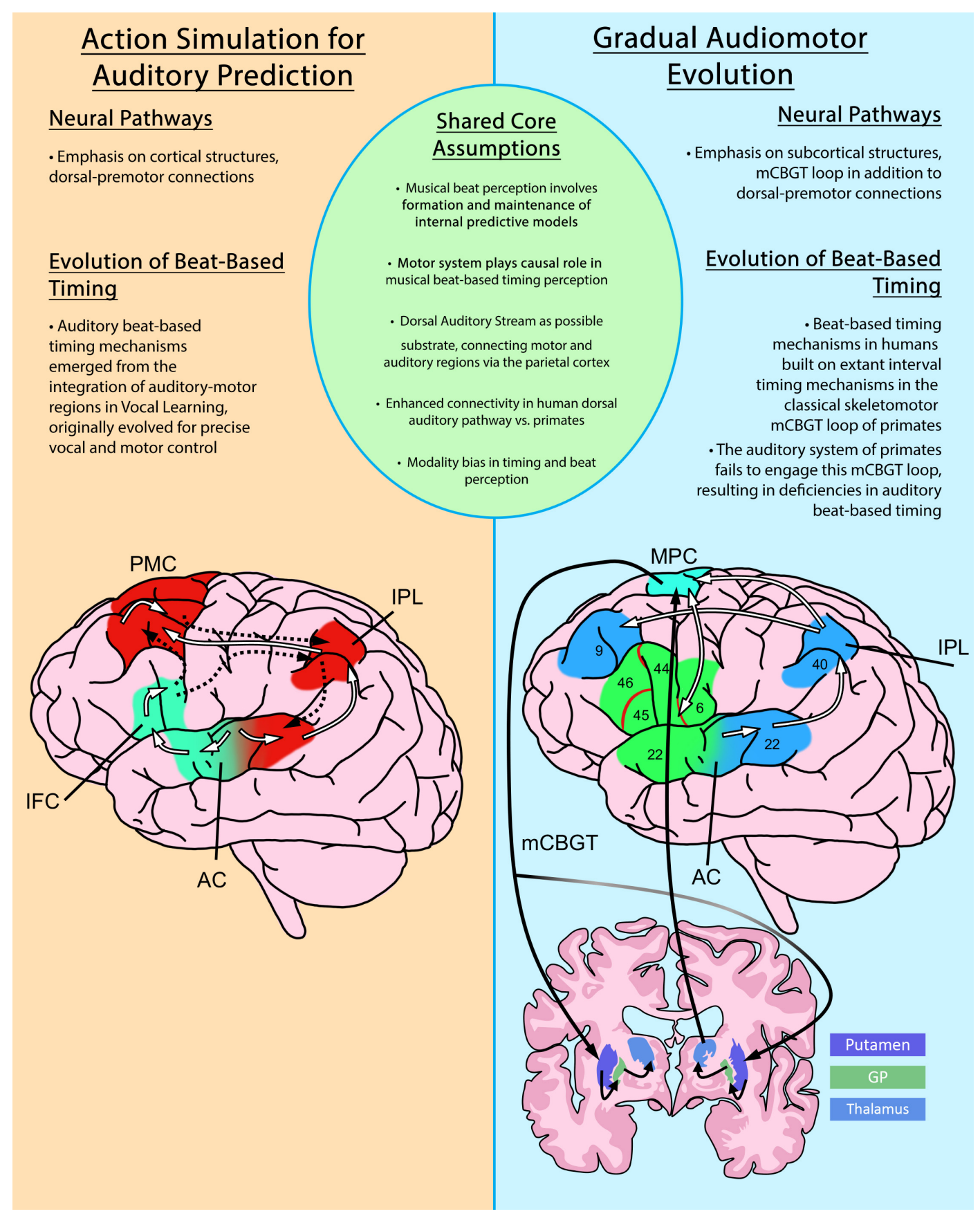

FIGURE 1 | An overview comparison of the Action Simulation for Auditory Prediction Hypothesis (ASAP) and the Gradual Audiomotor Evolution Hypothesis (GAE). Shared core assumptions of both hypotheses are listed at center. Brief differing emphases on neural pathways and evolutionary commitments are listed in each panel. Diagrams depict the neural pathways proposed under each hypothesis. The ASAP diagram (left), shows ascending pathways from the auditory cortex (white lines) and descending pathways back to the auditory cortex (dashed lines) in the dorsal (red) and ventral (green) streams. The GAE diagram (right) shows the dorsal auditory pathway (white lines) and dorsal (blue) and ventral (green) streams, and the motor cortico-basal ganglia-thalamo-cortical (mCBGT) circuit (black lines). PMC, primary motor cortex; IPL, inferior parietal lobule; AC, auditory cortex; IFC, inferior frontal cortex; MPC, medial premotor cortex; GP, globus pallidus. Figures adapted from Merchant and Honing (2014) and Patel and Iversen (2014).

adaptations to the motor cortico-basal ganglia thalamo-cortical circuit (mCBGT). This specification arises from observations that the mCBGT is found to be active in sequential and temporal processing and movement in Macaques (Tanji, 2001; Merchant et al., 2013; Perez et al., 2013) and humans (Grafton et al., 1995; Harrington et al., 2010), including, for humans, the processing of musical rhythms (Grahn and Brett, 2007). Explicitly including the mCBGT loop in the evolution of rhythmic entrainment accounts 
for the fact that interval-timing ability appears preserved in macaques (Merchant et al., 2013) and is shared among primates, including humans. This indicates a shared neural circuitry for single interval-based timing, upon which GAE hypothesizes human beat-based timing mechanisms would have evolved to enable beat-based rhythmic entrainment. It is gradual changes to this foundational neural pathway, in addition to strengthening connections to auditory cortices via the dorsal auditory pathway, that have enabled the human mCBGT to develop beat-based timing mechanisms that can process the hierarchical properties of beat-based, rhythmic stimuli, such as music. Although focusing on slightly different neural pathways, both ASAP and GAE highlight the predictive role of the motor system in the perception of rhythm, and support growing consensus on the role of motor pathways in the formation of internal predictive models in perception more generally.

One important difference between the ASAP and GAE hypotheses is that ASAP purports to explain the presence of motor activity in beat perception even in the absence of overt movement, while GAE explains how evolution within motor pathways enables physical entrainment-synchronized movement-to a rhythmic stimulus. ASAP claims that beatperception in humans arose with the emergence of vocal learning abilities, which strengthened tight audio-motor connections in the dorsal auditory stream underlying rhythmic entrainment along the primate lineage.

In contrast, GAE favors a gradual strengthening of these connections over evolutionary time, building on specific intervaltiming mechanisms already extant in the mCGBT circuit of the primate brain. The result being the formation of an additional beat-based mechanism with enhanced connection of the mCGBT to the auditory cortex via that same dorsal auditory stream in the human brain (Merchant and Honing, 2014). Recent neurophysiological evidence highlights the interconnectedness of interval and beat-based timing mechanisms proposed by GAE, indicating that even in passive listening, monkeys are able to detect isochrony in rhythm, due in part to extant interval-based timing mechanisms of the monkey motor system, but that monkeys cannot detect the underlying beat in a rhythmic stimulus, which requires auditory-motor beat-based timing mechanisms present in humans (Honing et al., 2018).

\section{Evidence for Prediction and Motor Activity in GAE and ASAP}

In addition to fMRI observation of motor activation in music listening and rhythm processing, the predictive and causal roles of specific motor structures highlighted by the ASAP and GAE hypotheses have been experimentally tested via electroencephalography (EEG) and transcranial magnetic stimulation (TMS). Specific Event Related Potentials (ERPs) relating to prediction errors evoked by rhythmic deviations in musical stimuli include the mismatch negativity (MMN) and P3a (Honing et al., 2018; Koelsch et al., 2019). These auditory event related components indicate violation of temporal expectations in oddball paradigms, with early responses related to bottom-up sensory processing and later responses reflecting top-down cortical processes (Garrido et al., 2007) and (perhaps conscious) attention to deviant stimuli (Sussman et al., 2003). EEG studies provide insight into the neural mechanisms of beat-perception while removing the limitations of behavioral response (Honing, 2012). The MMN and P3a components have been observed in response to rhythmic violations in adult humans, as well as infants and monkeys (Ladinig et al., 2009; Winkler et al., 2009; Honing et al., 2018). However more recent research in monkeys comparing ERPs in passive listening to jittered and isochronous stimuli with occasional deviants have demonstrated that monkeys might be able to detect isochrony in rhythm - which could rely on extant interval timing mechanisms in the primate brain; but not the beat - which relies on more evolved beat-based timing mechanisms, while humans are able to detect both isochrony and the beat (Bouwer et al., 2016; Honing et al., 2018). This collection of experiments supports the gradual evolution of beat-based timing mechanisms hypothesized by GAE.

Action Simulation for Auditory Prediction has been further supported by TMS research, demonstrating causal links between specific types of beat processing and regions of the dorsal auditory stream. A set of TMS experiments evaluated the role of the posterior parietal cortex (PPC), which is thought to serve as an interface for bidirectional communication between auditory and motor regions of the brain, and the dorsal pre-motor cortex (dPMC), which is also part of the dorsal auditory stream and is associated with movement planning and synchronization to auditory stimuli (Chen et al., 2009; Giovannelli et al., 2014). By down-regulating neural activity in left PPC according to the Huang et al. (2005) protocol, Ross et al. (2018b) showed that left PPC may be involved in one aspect of beat-based timing-phase shift detection-but not tempo detection or discrete interval discrimination. Ross et al. (2018a) down-regulated activity in left $\mathrm{dPMC}$, showing that left $\mathrm{dPMC}$ may be involved in tempo detection, but not phase shift detection or discrete interval discrimination. Additionally, measures of Motor Evoked Potentials (MEPs) in single pulse TMS over the motor cortex have indicated that musical groove modulates cortical excitability in the motor cortex. High levels of musical groove are characterized by syncopated rhythms, enhanced energy in the bass line, and the phenomenological property of 'wanting to move' with the music (Janata et al., 2012; Stupacher et al., 2013; Ross et al., 2016b). High-groove music has been shown to more strongly activate the motor system (resulting in higher MEPs) when compared with low-groove music (Stupacher et al., 2013). These results indicate the bidirectionality of auditory-motor interactions, as causally down-regulating activity in the motor cortex can impair auditory perception of aspects of musical rhythm, and varying degrees of rhythmic information in auditory stimuli (i.e., syncopation and bass frequencies in musical groove) can change aspects of motor cortical function.

\section{Mechanisms for Timing and Rhythm Prediction}

While there is growing consensus that the motor system is causally involved in timing and rhythm perception, and that the neural substrate includes cortical structures of the dorsal 
auditory stream and subcortical structures within the motorcortico basal ganglia thalamo-cortical loop, the specific neural mechanisms which enable timing and rhythm perception within these substrates remains an open question. For some years, cognitive scientists have been looking for how internal timing can be instantiated by patterns of temporal stimuli via, e.g., clock-based or oscillatory mechanisms (Povel and Essens, 1985; Large and Jones, 1999). Given the amount of neuroscientific evidence pointing to a distributed timing network in the brain (Buonomano, 2014), mechanisms of entrainment to patterns of temporal stimuli have received significant attention. The striatal beat frequency model was suggested to support a clockbased mechanism based on banks of oscillators (Matell and Meck, 2000, 2004). In contrast, Large et al. (2015) describe an oscillatory model of pulse perception called Neural Resonance Theory (NRT), which provides a plausible mechanism of adaptive entrainment and beat-based timing without requiring an internal clock mechanism. According to NRT, rhythmic stimuli are encoded in sensory networks which interact with motor networks thus entraining them to the pulse frequency. Neural entrainment is induced to the pulse, even when the rhythmic stimulus itself lacks physical information at the location of the pulse-such as silences found 'on the beat' within syncopated rhythmsdemonstrating the influence of top-down effects on pulse perception (Large et al., 2015; Tal et al., 2017). The cerebellum has also been shown to play a prominent role in absolute timing (Nozaradan et al., 2017)-but not beat-based timing-with proposed mechanisms including an oscillatory pacemaker based on regular oscillations found within the inferior olive (Ashe and Bushara, 2014), and a state-spaced based mechanism, in which the timing of a stimulus can be inferred from the state of a relevant cortical network over time (Buonomano, 2014). In various cortical areas, ramping activity of neural firing rates has been proposed as a mechanism for interval-based timing-where interval duration is encoded in the modulation of neural spiking thresholds or by varying the slope of ramping activity preceding threshold (Durstewitz, 2003). However, in the Macaque brain, ramping activity has also been implicated for relative timing in coordination with multidimensional state space models as part of a multilayer timing system involving two neural populations (Merchant et al., 2014). These two neural populations are differentially associated with absolute and relative timing, and are observed in the medial motor cortex, consistent with the proposed role of the motor system under the GAE hypothesis (Crowe et al., 2014; Merchant et al., 2014).

Continuous state-space models have also been proposed in Active Inference accounts for the generation of predictive models in action and sensory processing more generally, neurally mediated by the balance of pre- and post-synaptic activity (Friston et al., 2017) and neuronal firing rates in, e.g., medial or lateral intraparietal areas (de Lafuente et al., 2015). Striatal dopamine in particular has been proposed to code for both prediction error and certainty in response to sensory stimuli (Sarno et al., 2017) across a variety of timescales (Schultz, 2007). Dopaminergic activity also plays a role in rhythmic motor control (Koshimori and Thaut, 2018) and is responsive to rhythmic auditory stimulation (Koshimori et al., 2019), positioning dopamine as a crucial facilitator of the motor system's role in auditory-motor interactions underlying beatbased timing perception. The motor system's predictive role in music and rhythm perception is only one component of larger networks of sensorimotor processing, namely the dorsal auditory pathway and the mCBGT. Further experimental and computational work is necessary to determine whether and how the specific neural mechanisms of the human motor cortex processes timing information within the cortical and subcortical networks proposed by ASAP and GAE. To facilitate the generation of experimental and computational hypotheses, we have compiled an overview of recent experimental and theoretical research on the motor and distributed brain areas and mechanisms within the dorsal auditory pathway and the mCBGT-including the dopaminergic system-which are involved in the predictive processing of auditory-motor beat and rhythm perception in Table 1.

\section{CONCLUSION AND FUTURE DIRECTIONS}

In this paper, we reviewed recent advances in research on rhythm perception, focusing on the role of predictive processes in auditory motor interactions in beat-processing. We highlighted two complementary hypotheses for the neural underpinnings of rhythm perception: The ASAP hypothesis (Patel and Iversen, 2014) and the GAE hypothesis (Merchant and Honing, 2014) and reviewed recent experimental progress supporting each of these hypotheses. While initial formulations of ASAP and GAE explain different aspects of beat-based timing-the involvement of motor structures in the absence of movement, and physical entrainment to an auditory beat respectively-both theories have moved us closer to understanding the predictive role of the motor system in the perception of rhythm and the specific neural mechanisms involved. In fact, recent computational formulations of ASAP have further incorporated the subcortical structures proposed to be involved in the evolution of beat-based timing perception by GAE. Cannon and Patel (2019, preprint), have proposed the CBGT loop as responsible for the resetting of relative timing mechanisms via a hyper direct pathway from the SMA. In addition, they hypothesize a role for striatal dopamine in the maintenance of internal rhythmic timing models by tracking confidence (uncertainty) in the beat, consistent with Predictive Coding and Active Inference accounts of rhythm perception and perception more generally.

Future work in understanding the neural, cognitive, and behavioral dynamics of musical beat perception in humans should investigate not only the sensorimotor processes responsible for the perception of rhythm, but also the specific neural mechanisms by which top-down predictions serve to modulate driving proprioceptive sensations arising from concrete actions of the body or abstract activity of the motor systems. While EEG experiments (e.g., Ladinig et al., 2009; Winkler et al., 2009; Honing et al., 2018) point to the neural mechanisms of internal predictive models in beat-based timing perception, EEG alone cannot provide causal evidence for 
TABLE 1 | The motor system's predictive role in music and rhythm perception is only one component of larger networks of sensorimotor processing, namely the dorsal auditory pathway and the motor cortico-basal ganglia-thalamo-cortical circuit.

\begin{tabular}{|c|c|c|c|c|}
\hline Brain area & Authors & Proposed role of each brain area & Experimental task and stimulus type & Type of data \\
\hline \multirow[t]{4}{*}{ Cerebellum } & Ivry and Schlerf, 2008 & $\begin{array}{l}\text { Dedicated timing mechanism; coordination of movement, } \\
\text { internal timing mechanisms involved with sub-second } \\
\text { timing }\end{array}$ & Theoretical Paper/Review & \\
\hline & Bastian, 2006 & Predictive models of movement & Theoretical Paper/Review & \\
\hline & Nozaradan et al., 2017 & $\begin{array}{l}\text { Tracking beats in rhythms with fast tempos; more } \\
\text { prominent role in absolute timing vs. relative timing }\end{array}$ & $\begin{array}{l}\text { Passive Listening. Auditory rhythms designed to induce a } \\
\text { beat - syncopated and unsyncopated. }\end{array}$ & EEG \\
\hline & Gordon et al., 2018 & $\begin{array}{l}\text { Meta-analysis of fMRI studies of recruitment of motor } \\
\text { system during music listening }\end{array}$ & $\begin{array}{l}\text { Meta-analysis. Various listening tasks - auditory rhythms } \\
\text { or music. }\end{array}$ & fMRI \\
\hline \multirow[t]{9}{*}{ Basal Ganglia } & Merchant et al., 2013 & $\begin{array}{l}\text { Interacts with the cortico-thalamic-striatal circuit in a } \\
\text { context dependent manner }\end{array}$ & Theoretical Paper/Review & \\
\hline & Coull and Nobre, 2008 & Perceptual temporal expectation; explicit timing & Theoretical Paper/Review & \\
\hline & Nozaradan et al., 2017 & Tracking beats in complex rhythm sequences & $\begin{array}{l}\text { Passive Listening Auditory rhythms designed to induce a } \\
\text { beat - syncopated and unsyncopated. }\end{array}$ & EEG \\
\hline & Grahn, 2009 & $\begin{array}{l}\text { Internal beat generation; more prominent role in relative vs. } \\
\text { absolute timing }\end{array}$ & $\begin{array}{l}\text { Discrimination task, same or different judgment of } \\
\text { two auditory stimuli. Auditory rhythms - beat-based } \\
\text { structure and non-beat-based structure; Accents- duration } \\
\text { or volume accented (externally generated) or unaccented } \\
\text { (internally generated) beats. }\end{array}$ & fMRI/Behaviora \\
\hline & Grahn et al., 2011 & $\begin{array}{l}\text { Internal representation of auditory rhythms that support } \\
\text { cross-modal interactions in beat perception and generation }\end{array}$ & $\begin{array}{l}\text { Discrimination task, rhythmic tempo change. Auditory } \\
\text { tone metronome and visual flashing metronome. Two } \\
\text { groups: one with auditory first visual second, and the other } \\
\text { vice versa. }\end{array}$ & fMRI/Behaviora \\
\hline & Grahn and Rowe, 2009 & $\begin{array}{l}\text { Internal beat generation: part of cortico-subcortical network } \\
\text { involved in beat perception and generation }\end{array}$ & $\begin{array}{l}\text { Indicate the strength of the perceived beat. Auditory } \\
\text { rhythms of varying complexity and some with volume } \\
\text { accents. }\end{array}$ & fMRI/Behaviora \\
\hline & Grahn and Rowe, 2013 & Putamen activity in beat prediction, but not beat finding & $\begin{array}{l}\text { Attentive listening; occasionally indicate level of } \\
\text { feeling of the beat. Auditory rhythms of varying intervals } \\
\text { and rates, beat and non-beat (jittered) rhythms. }\end{array}$ & fMRI/Behaviora \\
\hline & Grahn and Brett, 2007 & $\begin{array}{l}\text { Higher activity for rhythms with integer ratio relationships } \\
\text { between intervals and with regular perceptual accents }\end{array}$ & $\begin{array}{l}\text { 1st experiment (behavioral) reproduce auditory rhythms. } \\
\text { 2nd experiment (fMRI) indicate if the rhythm played } \\
\text { matched previous rhythms. Metered auditory rhythms of } \\
\text { varying integer intervals and complexity. }\end{array}$ & fMRI/Behaviora \\
\hline & Teki et al., 2011 & $\begin{array}{l}\text { Striato-thalamo-cortical network involved in beat-based } \\
\text { timing, while an olivocerebellar network involved in } \\
\text { duration-based timing }\end{array}$ & $\begin{array}{l}\text { Judge duration matches in a set of tones. Auditory } \\
\text { tones, either isochronous or jittered, arranged in either } \\
\text { rhythm-based or absolute duration-based sets. }\end{array}$ & $\mathrm{fMRI} /$ Behaviora \\
\hline
\end{tabular}


TABLE 1 | Continued

\begin{tabular}{|c|c|c|c|c|}
\hline Brain area & Authors & Proposed role of each brain area & Experimental task and stimulus type & Type of data \\
\hline & Araneda et al., 2017 & $\begin{array}{l}\text { Hearing, feeling or seeing a beat recruits a supramodal } \\
\text { network in the auditory dorsal stream }\end{array}$ & $\begin{array}{l}\text { Discrimination task, between beat and non-beat } \\
\text { rhythms. Auditory, visual, and vibrotactile rhythms. }\end{array}$ & fMRI/Behavioral \\
\hline $\begin{array}{l}\text { Primary and } \\
\text { premotor }\end{array}$ & Kilavik et al., 2014 & Movement preparation, cue anticipation & Theoretical Paper/Review & \\
\hline
\end{tabular}

premotor

Schubotz, 2007

Morillon and Baillet, 2017

Gordon et al., 2018

Premotor

cortex

$\begin{aligned} & \text { Supplementary Coull et al., 2016b } \\ & \text { motor area }\end{aligned}$
noss eta., $2018 b$

Ross et al., 2018b

Grahn and Brett, 2007

Grahn and McAuley, 2009
Predictive processing of external events, even in the absence of proprioceptive or interoceptive information

Beta and delta oscillations directed to auditory cortex encode temporal predictions

Meta-analysis of fMRI studies of recruitment of motor system during music listening

Cortico-cortical coupling with SMA and auditory cortex in duration beat perception; part of cortico-subcortical network involved in beat perception and generation Striato-thalamo-cortical network involved in beat-based timing, while an olivocerebellar network involved in duration based timing

Motor regions recruited while listening to music rhythms

Perceptual and motor timing; Comparing the duration of perceptual events, error monitoring

Not causally implicated in perceptual auditory interval timing

Higher activity for rhythms with integer ratio relationships between intervals and with regular perceptual accents; in musicians: higher activity for all rhythms when compared to rest

Stronger activity in strong beat-perceivers vs. weak beat-perceivers, no correlation with musicianship
Theoretical Paper/Review

Passive listening (listen condition); active tapping

with the beat (tracking condition). Auditory melody different tones either on beat, anti-phase, or quasi-phase with the beat.

Meta-analysis. Various listening tasks - Auditory rhythms or music.

Indicate the strength of the perceived beat. Auditor

rhythms of varying complexity and some with volume accents.

Judge duration matches in a set of tones. Auditory ones, either isochronous or jittered, arranged in either

hythm-based or absolute duration-based sets.

Experiment 1: Listen to rhythm passively then tap along with rhythm. Experiment 2: Listen to rhythm passively then tap along to rhythm without

foreknowledge of being asked to tap with the rhythm auditory tones in simple, complex, or ambiguous rhythms.

Theoretical Paper/Review

Discrimination task - same/different judgment of auditory intervals; detection task - identification of tempo or phase shifted metronome click. Auditory intervals of pairs of tones; metronome click track over musical stimuli.

Experiment 1 (behavioral): reproduce auditory rhythms. Experiment 2 (fMRI): indicate if the rhythm played matched previous rhythms. Metered auditory rhythms of varying integer intervals and complexity.

Discrimination task, rhythmic tempo change. Auditory isochronous rhythms.
MEG/Behavioral

fMR

fMRI/Behavioral

fMRI/Behavioral

fMRI/Behavioral

Behavioral (pre/post TMS down-regulatory stimulation)

fMRI/Behavioral

fMRI/Behavioral 
TABLE 1 | Continued

\begin{tabular}{lll}
\hline Brain area & Authors & Proposed role of each brain area \\
\hline Grahn and Rowe, 2009 & $\begin{array}{l}\text { Coupling with STG in beat perception for musicians; part of } \\
\text { cortico-subcortical network involved in beat perception and } \\
\text { generation }\end{array}$ \\
& $\begin{array}{l}\text { Striato-thalamo-cortical network involved in beat-based } \\
\text { time, while an olivocerebellar network involved in } \\
\text { Teki et al., 2011 }\end{array}$ \\
& duration-based timing \\
& Motor regions recruited while listening to music rhythms
\end{tabular}

\section{Experimental task and stimulus type}

network in the auditory dorsal stream

Absolute and relative timing mechanisms within two

separate neural populations

premotor

Merchant et al., 2014

cortex

Crowe et al., 2014

Grahn and McAuley, 2009

Absolute and relative timing

Stronger activity in strong beat-perceivers vs. weak beat-perceivers, no correlation with musicianship

Interface between motor and auditory cortices, sensorimotor integration

Merchant and Honing, 2014; Patel and Iversen, 2014

Coull and Nobre, 2008

Coull et al., 2016a

Ross et al., 2018b
Perceptual temporal expectation; implicit timing

Temporal predictability via fixed or dynamic predictions

Causally implicated in perceptual beat-based timing
Indicate the strength of the perceived beat. Auditor

rhythms of varying complexity and some with volume

accents.

Judge duration matches in a set of tones. Auditory

tones, either isochronous or jittered, arranged in either

rhythm-based or absolute duration-based sets.

Experiment 1: Listen to rhythm passively then tap along with rhythm. Experiment 2: Listen to rhythm passively then tap along to rhythm without

foreknowledge of being asked to tap with the rhythm

auditory tones in simple, complex, or ambiguous rhythms.

Discrimination task, between beat and non-beat

rhythms. Auditory, visual, and vibrotactile rhythms.

\section{Type of data}

fMRI/Behavioral

fMRI/Behavioral

fMRI/Behavioral

fMRI/Behavioral

Theoretical Paper/Review

Synchronization Continuation Task. Isochronous visual stimuli or auditory tones.

Discrimination task, rhythmic tempo change. Auditory sochronous rhythms.

Theoretical Papers/Reviews

Theoretical Paper/Review

Cued reaction time task. Visual cue that predicted target presentation time (temporal condition), or provided no

information for target presentation (neutral condition) with variable intervals between cue and target.

Discrimination task - same/different judgment of auditory intervals; detection task - identification of

tempo or phase shifted metronome click. Auditory

intervals of pairs of tones: metronome click track over

musical stimuli.
Behavioral; Extracellular activity of single neurons (in Macaca mulatta)

fMRI/Behavioral

\section{fMRI/Behavioral}

Behavioral (pre/post TMS down-regulatory

stimulation) 
TABLE 1 | Continued

\begin{tabular}{|c|c|c|c|c|}
\hline Brain area & Authors & Proposed role of each brain area & Experimental task and stimulus type & Type of data \\
\hline \multirow[t]{6}{*}{ Auditory Cortex } & Koelsch et al., 2019 & $\begin{array}{l}\text { Event related potentials associated with predictive } \\
\text { processes in music }\end{array}$ & Theoretical Paper/Review & \\
\hline & Fujioka et al., 2012 & Beta-band activity predicts onset of beats in music & $\begin{array}{l}\text { Passive listening, while watching silent videos. } \\
\text { Auditory isochronous rhythms of several tempos and one } \\
\text { irregular rhythm. }\end{array}$ & MEG \\
\hline & Fujioka et al., 2015 & $\begin{array}{l}\text { Beta-band activity represents timing information being } \\
\text { translated for auditory-motor coordination }\end{array}$ & $\begin{array}{l}\text { Passive listening to metered rhythms, followed by } \\
\text { attentive listening to un-metered rhythms that the } \\
\text { participants were asked to imagine as metered. March and } \\
\text { Waltz metered rhythms }\end{array}$ & MEG \\
\hline & Auksztulewicz et al., 2019 & Temporal prediction of rhythm and beats & $\begin{array}{l}\text { Identify target chords. Auditory rhythmic or jittered } \\
\text { sequences of distractor chords preceding target chords. }\end{array}$ & MEG/EEG/Behavioral \\
\hline & Honing et al., 2018 & $\begin{array}{l}\text { Event related potentials to perceptual deviants in rhythmic } \\
\text { stimuli }\end{array}$ & $\begin{array}{l}\text { Passive listening. Auditory oddball paradigm with } \\
\text { isochronous or jittered rhythms. }\end{array}$ & EEG (of Macaca mulatta) \\
\hline & Bouwer et al., 2016 & $\begin{array}{l}\text { Event related potentials to perceptual deviants in rhythmic } \\
\text { stimuli; ERPs modulated by attention in musicians }\end{array}$ & $\begin{array}{l}\text { Passive or attentive listening. Auditory oddball paradigm } \\
\text { with isochronous or jittered rhythms. }\end{array}$ & EEG \\
\hline \multirow{6}{*}{$\begin{array}{l}\text { Dopaminergic } \\
\text { System/Striatal } \\
\text { Dopamine }\end{array}$} & Schultz, 2007 & $\begin{array}{l}\text { Multiple time courses of dopamine changes mediate } \\
\text { multiple time courses of behavioral processes }\end{array}$ & Theoretical Paper/Review & \\
\hline & Friston et al., 2009 & Reward learning, encoding of precision & Theoretical Paper/Review & \\
\hline & $\begin{array}{l}\text { Friston et al., 2012; } \\
\text { FitzGerald et al., } 2015\end{array}$ & Reward learning, encoding of precision & Theoretical Papers/Computational Models & $\begin{array}{l}\text { Simulated dopaminergic } \\
\text { responses }\end{array}$ \\
\hline & Sarno et al., 2017 & $\begin{array}{l}\text { Temporal expectation of perceptual cues; reward prediction } \\
\text { error and (un)certainty }\end{array}$ & $\begin{array}{l}\text { Detect weak vibrotactile stimuli. Variable interval } \\
\text { durations between tactile start cue and vibrotactile stimuli. }\end{array}$ & $\begin{array}{l}\text { Intracellular recording, } \\
\text { monkey brain }\end{array}$ \\
\hline & Koshimori et al., 2019 & $\begin{array}{l}\text { Rhythmic auditory stimulation (RAS) attenuates } \\
\text { dopaminergic response }\end{array}$ & $\begin{array}{l}\text { Synchronization task, RAS and no-RAS conditions; } \\
\text { various auditory rhythms, single auditory beats or } \\
\text { metronome clicks over instrumental music. }\end{array}$ & Behavioral/MRI/PET \\
\hline & Brodal et al., 2017 & $\begin{array}{l}\text { Rhythmic music reduces connectivity between basal } \\
\text { ganglia and reward system }\end{array}$ & $\begin{array}{l}\text { Passive listening. Electronic dance music in a } \\
\text { continuous-stimulation design. }\end{array}$ & fMRI \\
\hline
\end{tabular}

This table provides an overview of the brain areas and mechanisms which make up these networks and are involved in the predictive processing of auditory beat and rhythm perception. Each brain area is introduced with one or more Theoretical or Review Papers contextualizing that brain area's proposed role, followed by a non-exhaustive list of supporting experimental work. This table is intended to serve as a tool for new or continuing researchers engaging in work on rhythm and musical beat perception. 
the role of specific brain structures. Similarly, while TMS experiments (e.g., Stupacher et al., 2013; Ross et al., 2018a,b) have lended causal evidence for the role of specific structures in beat-based timing perception, the mentioned experiments do not provide direct evidence for the presence of internal predictive models of beat-based timing. If motor activity is causally involved in the formation of auditory predictions, then causal TMS manipulation to down-regulate activity in, e.g., parietal cortex or $\mathrm{APMC}$ should result in the reduction of MMN and P3a event related responses to perceptual deviants in rhythmic stimuli, and this response might differ based on whether the stimuli contains timing deviants related to tempo or phase. Future research should include stimuli designed to elicit specific prediction errors with perceptual deviants, such as

\section{REFERENCES}

Adams, R. A., Shipp, S., and Friston, K. J. (2013). Predictions not commands: active inference in the motor system. Brain Struct. Funct. 218, 611-643. doi: 10.1007/s00429-012-0475-5

al-Haytham, I. A. (ca. 1030; 1989). The Optics of Ibn al-Haytham. Translated by A. I. Sabra. London: Warburg Institute.

Araneda, R., Renier, L., Ebner-Karestinos, D., Dricot, L., and De Volder, A. G. (2017). Hearing, feeling or seeing a beat recruits a supramodal network in the auditory dorsal stream. Eur. J. Neurosci. 45, 1439-1450. doi: 10.1111/ejn.13349

Ashe, J., and Bushara, K. (2014). The olivo-cerebellar system as a neural clock. Adv. Exp. Med. Biol. 829, 155-165. doi: 10.1007/978-1-4939-1782-2_9

Auksztulewicz, R., Myers, N. E., Schnupp, J. W., and Nobre, A. C. (2019). Rhythmic temporal expectation boosts neural activity by increasing neural gain. J. Neurosci. 39, 9806-9817. doi: 10.1523/jneurosci.0925-19.2019

Balasubramaniam, R., Wing, A., and Daffertshofer, A. (2004). Keeping with the beat: movement trajectories contribute to movement timing. Exp. Brain Res. 159, 129-134. doi: 10.1007/s00221-004-2066-z

Bastian, A. J. (2006). Learning to predict the future: the cerebellum adapts feedforward movement control. Curr. Opin. Neurobiol. 16, 645-649. doi: 10. 1016/j.conb.2006.08.016

Bouwer, F. L., Werner, C. M., Knetemann, M., and Honing, H. (2016). Disentangling beat perception from sequential learning and examining the influence of attention and musical abilities on ERP responses to rhythm. Neuropsychologia 85, 80-90. doi: 10.1016/j.neuropsychologia.2016.02.018

Brodal, H. P., Osnes, B., and Specht, K. (2017). Listening to rhythmic music reduces connectivity within the basal ganglia and the reward system. Front. Neurosci. 11:153. doi: 10.3389/fnins.2017.00153

Bruineberg, J., Kiverstein, J., and Rietveld, E. (2018). The anticipating brain is not a scientist: the free-energy principle from an ecologicalenactive perspective. Synthese 195, 2417-2444. doi: 10.1007/s11229-0161239-1

Buonomano, D. V. (2014). Neural dynamics based timing in the subsecond to seconds range. Adv. Exp. Med. Biol. 829, 101-117. doi: 10.1007/978-1-49391782-2_6

Cameron, D. J., Bentley, J., and Grahn, J. A. (2015). Cross-cultural influences on rhythm processing: reproduction, discrimination, and beat tapping. Front. Psychol. 6:366. doi: 10.3389/fpsyg.2015.00366

Cannon, J. J., and Patel, A. D. (2019). A neurocomputational model of beat-based temporal processing. bioRxiv [Preprint] doi: 10.1101/805838

Chen, J. L., Penhune, V. B., and Zatorre, R. J. (2008a). Listening to musical rhythms recruits motor regions of the brain. Cereb. Cortex 18, 2844-2854. doi: $10.1093 /$ cercor/bhn042

Chen, J. L., Penhune, V. B., and Zatorre, R. J. (2008b). Moving on time: brain network for auditory-motor synchronization is modulated by rhythm complexity and musical training. J. Cogn. Neurosci. 20, 226-239. doi: 10.1162/ jocn.2008.20018 in oddball paradigms, while measuring event-related potentials associated with predictive processes in combined EEG and causal TMS experiments. Results from these experiments could extend and strengthen already emerging support for GAE and ASAP, as well as further contextualize the role of Active Inference in music and beat-based timing perception.

\section{AUTHOR CONTRIBUTIONS}

SP and RB conceptualized the manuscript. BM, DC, and AP contributed to the writing and the exhaustive analysis of the literature. All authors contributed to the article and approved the submitted version.

Chen, J. L., Penhune, V. B., and Zatorre, R. J. (2009). The role of auditory and premotor cortex in sensorimotor transformations. Ann. N. Y. Acad. Sci. 1169, 15-34. doi: 10.1111/j.1749-6632.2009.04556.x

Clark, A. (2013). Whatever next? Predictive brains, situated agents, and the future of cognitive science. Behav. Brain Sci. 36, 181-204. doi: 10.1017/ S0140525X12000477

Coull, J. T., Cotti, J., and Vidal, F. (2016a). Differential roles for parietal and frontal cortices in fixed versus evolving temporal expectations: Dissociating prior from posterior temporal probabilities with fMRI. NeuroImage 141, 40-51. doi: 10.1016/j.neuroimage.2016.07.036

Coull, J. T., Vidal, F., and Burle, B. (2016b). When to act, or not to act: that's the SMAs question. Curr. Opin. Behav. Sci. 8, 14-21. doi: 10.1016/j.cobeha.2016.01. 003

Coull, J. T., and Nobre, A. C. (2008). Dissociating explicit timing from temporal expectation with fMRI. Curr. Opin. Neurobiol. 18, 137-144. doi: 10.1016/j.conb. 2008.07.011

Crowe, D. A., Zarco, W., Bartolo, R., and Merchant, H. (2014). Dynamic representation of the temporal and sequential structure of rhythmic movements in the primate medial premotor cortex. J. Neurosci. 34, 11972-11983. doi: 10.1523/jneurosci.2177-14.2014

de Lafuente, V., Jazayeri, M., and Shadlen, M. N. (2015). Representation of accumulating evidence for a decision in two parietal areas. J. Neurosci. 35, 4306-4318. doi: 10.1523/jneurosci.2451-14.2015

Durstewitz, D. (2003). Self-organizing neural integrator predicts interval times through climbing activity. J. Neurosci. 23, 5342-5353. doi: 10.1523/jneurosci. 23-12-05342.2003

FitzGerald, T. H. B., Dolan, R. J., and Friston, K. (2015). Dopamine, reward learning, and active inference. Front. Comput. Neurosci. 9:136. doi: 10.3389/ fncom.2015.00136

Friston, K. (2002). Beyond phrenology: what can neuroimaging tell us about distributed circuitry? Annu. Rev. Neurosci. 25, 221-250. doi: 10.1146/annurev. neuro.25.112701.142846

Friston, K. (2005). A theory of cortical responses. Philos. Trans. R. Soc. B Biol. Sci. 360, 815-836. doi: 10.1098/rstb.2005.1622

Friston, K. (2010). The free-energy principle: a unified brain theory? Nat. Rev. Neurosci. 11, 127-138. doi: 10.1038/nrn2787

Friston, K., and Stephan, K. E. (2007). Free-energy and the brain. Synthese 159, 417-458. doi: 10.1007/s11229-007-9237-y

Friston, K. J., Daunizeau, J., and Kiebel, S. J. (2009). Reinforcement learning or active inference? PLoS One 4:7. doi: 10.1371/journal.pone.0006421

Friston, K. J., and Friston, D. A. (2013). "A free energy formulation of music generation and perception: Helmholtz revisited," in Sound - Perception Performance. Current Research in Systematic Musicology, Vol. 1, ed. R. Bader (Heidelberg: Springer), 43-69. doi: 10.1007/978-3-319-00107-4_2

Friston, K. J., Parr, T., and de Vries, B. (2017). The graphical brain: Belief propagation and active inference. Net. Neurosci. 1, 381-414. doi: 10.1162/netn a_00018 
Friston, K. J., Shiner, T., Fitzgerald, T., Galea, J. M., Adams, R., Brown, H., et al. (2012). Dopamine, affordance and active inference. PLoS Comput. Biol. 8:e1002327. doi: 10.1371/journal.pcbi.1002327

Fujioka, T., Ross, B., and Trainor, L. J. (2015). Beta-band oscillations represent auditory beat and its metrical hierarchy in perception and imagery. J. Neurosci. 35, 15187-15198. doi: 10.1523/JNEUROSCI.2397-15.2015

Fujioka, T., Trainor, L. J., Large, E. W., and Ross, B. (2012). Internalized timing of isochronous sounds is represented in neuromagnetic beta oscillations. J. Neurosci. 32, 1791-1802. doi: 10.1523/jneurosci.4107-11. 2012

Gallagher, S., Hutto, D. D., Slaby, J., and Cole, J. (2013). The brain as part of an enactive system. Behav. Brain Sci. 36, 421-422. doi: 10.1017/ S0140525X12002105

Garrido, M. I., Kilner, J. M., Kiebel, S. J., and Friston, K. J. (2007). Evoked brain responses are generated by feedback loops. Proc. Natl. Acad. Sci. U.S.A. 104, 20961-20966. doi: 10.1073/pnas.0706274105

Giovannelli, F., Innocenti, I., Rossi, S., Borgheresi, A., Ragazzoni, A., Zaccara, G., et al. (2014). Role of the dorsal premotor cortex in rhythmic auditory-motor entrainment: a perturbational approach by rTMS. Cereb. Cortex 24, 1009-1016. doi: $10.1093 /$ cercor/bhs386

Gordon, C. L., Cobb, P. R., and Balasubramaniam, R. (2018). Recruitment of the motor system during music listening: an ALE meta-analysis of fMRI data. PLos One 13:e0207213. doi: 10.1371/journal.pone.0207213

Grafton, S. T., Hazeltine, E., and Ivry, R. (1995). Functional mapping of sequence learning in normal humans. J. Cogn. Neurosci. 7, 497-510. doi: 10.1162/jocn. 1995.7.4.497

Grahn, J. A. (2009). The role of the basal ganglia in beat perception. Ann. N. Y. Acad. Sci. 1169, 35-45. doi: 10.1111/j.1749-6632.2009.04553.x

Grahn, J. A., and Brett, M. (2007). Rhythm and beat perception in motor areas of the brain. J. Cogn. Neurosci. 19, 893-906. doi: 10.1162/jocn.2007.19.5.893

Grahn, J. A., Henry, M. J., and McAuley, J. D. (2011). FMRI investigation of crossmodal interactions in beat perception: audition primes vision, but not vice versa. NeuroImage 54, 1231-1243. doi: 10.1016/j.neuroimage.2010.09.033

Grahn, J. A., and McAuley, J. D. (2009). Neural bases of individual differences in beat perception. NeuroImage 47, 1894-1903. doi: 10.1016/j.neuroimage.2009. 04.039

Grahn, J. A., and Rowe, J. B. (2009). Feeling the beat: premotor and striatal interactions in musicians and nonmusicians during beat perception. J. Neurosci. 29, 7540-7548. doi: 10.1523/jneurosci.2018-08.2009

Grahn, J. A., and Rowe, J. B. (2013). Finding and feeling the musical beat: striatal dissociations between detection and prediction of regularity. Cereb. Cortex 23, 913-921. doi: 10.1093/cercor/bhs083

Grube, M., Lee, K.-H., Griffiths, T. D., Barker, A. T., and Woodruff, P. W. (2010). Transcranial magnetic theta-burst stimulation of the human cerebellum distinguishes absolute, duration-based from relative, beat-based perception of subsecond time intervals. Front. Psychol. 1:171. doi: 10.3389/fpsyg.2010.00171

Harrington, D. L., Zimbelman, J. L., Hinton, S. C., and Rao, S. M. (2010). Neural modulation of temporal encoding, maintenance, and decision processes. Cereb. Cortex 20, 1274-1285. doi: 10.1093/cercor/bhp194

Hohwy, J. (2013). The Predictive Mind. New York, NY: Oxford University Press.

Honing, H. (2012). Without it no music: beat induction as a fundamental musical trait. Ann. N. Y. Acad. Sci. 1252, 85-91. doi: 10.1111/j.1749-6632.2011. 06402.x

Honing, H., Bouwer, F. L., and Háden, G. P. (2014). “Perceiving temporal regularity in music: the role of auditory event-related potentials (ERPs) in probing beat perception," in Neurobiology of Interval Timing. Advances in Experimental Medicine and Biology, eds H. Merchant and V. de Lafuente (New York, NY: Springer), 305-323. doi: 10.1007/978-1-4939-1782-2_16

Honing, H., Bouwer, F. L., Prado, L., and Merchant, H. (2018). Rhesus monkeys (Macaca mulatta) sense isochrony in rhythm, but not the beat: additional support for the gradual audiomotor evolution hypothesis. Front. Neurosci. 12:475. doi: 10.3389/fnins.2018.00475

Hove, M. J., and Keller, P. E. (2010). Spatiotemporal relations and movement trajectories in visuomotor synchronization. Music Percept. 28, 15-26. doi: 10. 1525/mp.2010.28.1.15

Huang, Y. Z., Edwards, M. J., Rounis, E., and Rothwell, J. C. (2005). Theta burst stimulation of the human motor cortex. Neuron 45, 201-206. doi: 10.1016/j. neuron.2004.12.033
Huron, D. (2008). Sweet Anticipation: Music and the Psychology of Expectation. Cambridge, MA: MIT Press.

Iversen, J. R., and Balasubramaniam, R. (2016). Synchronization and temporal processing. Curr. Opin. Behav. Sci. 8, 175-180. doi: 10.1016/j.cobeha.2016.02. 027

Iversen, J. R., Patel, A. D., Nicodemus, B., and Emmorey, K. (2015). Synchronization to auditory and visual rhythms in hearing and deaf individuals. Cognition 134, 232-244. doi: 10.1016/j.cognition.2014.10.018

Ivry, R. B., and Schlerf, J. E. (2008). Dedicated and intrinsic models of time perception. Trends Cogn. Sci. 12, 273-280. doi: 10.1016/j.tics.2008.04.002

Jacoby, N., and McDermott, J. H. (2017). Integer ratio priors on musical rhythm revealed cross-culturally by iterated reproduction. Curr. Biol. 27, 359-370. doi: 10.1016/j.cub.2016.12.031

Janata, P., Tomic, S. T., and Haberman, J. M. (2012). Sensorimotor coupling in music and the psychology of the groove. J. Exp. Psychol. 141, 54-75. doi: 10. 1037/a0024208

Juslin, P. N., and Västfjäll, D. (2008). Emotional responses to music: the need to consider underlying mechanisms. Behav. Brain Sci. 31, 751-751. doi: 10.1017/ s0140525x08006079

Kilavik, B. E., Confais, J., and Riehle, A. (2014). Signs of timing in motor cortex during movement preparation and cue anticipation. Adv. Exp. Med. Biol. 829, 121-142. doi: 10.1007/978-1-4939-1782-2_7

Koelsch, S., Vuust, P., and Friston, K. (2019). Predictive processes and the peculiar case of music. Trends Cogn. Sci. 23, 63-77. doi: 10.1016/j.tics.2018.10.006

Koshimori, Y., Strafella, A. P., Valli, M., Sharma, V., Cho, S.-S., Houle, S., et al. (2019). Motor synchronization to rhythmic auditory stimulation (RAS) attenuates dopaminergic responses in ventral striatum in young healthy adults: [11C]-()-PHNO PET study. Front. Neurosci. 13:106. doi: 10.3389/fnins.2019. 00106

Koshimori, Y., and Thaut, M. H. (2018). Future perspectives on neural mechanisms underlying rhythm and music based neurorehabilitation in Parkinson's disease. Aging Res. Rev. 47, 133-129. doi: 10.1016/j.arr.2018.07.001

Kotz, S. A., Ravignani, A., and Fitch, T. C. (2018). The evolution of rhythm processing. Trends Cogn. Sci. 10, 896-910. doi: 10.1016/j.tics.2018.08.002

Ladinig, O., Honing, H., Háden, G., and Winkler, I. (2009). Probing attentive and pre-attentive emergent meter in adult listeners without extensive music training. Music Percept. 28, 377-386. doi: 10.1525/mp.2009.26.4.377

Large, E. W., Herrera, J. A., and Velasco, M. J. (2015). Neural networks for beat perception in musical rhythm. Front. Syst. Neurosci. 9:159. doi: 10.3389/fnsys. 2015.00159

Large, E. W., and Jones, M. R. (1999). The dynamics of attending: How people track time-varying events. Psychol. Rev. 106, 119-159. doi: 10.1037/0033-295X.106.1. 119

Matell, M. S., and Meck, W. H. (2000). Neuropsychological mechanisms of interval timing behavior. Bioessays 22, 94-103. doi: 10.1002/(SICI)1521-1878(200001) 22:1<94::AID-BIES14<3.0.CO;2-E

Matell, M. S., and Meck, W. H. (2004). Cortico-striatal circuits and interval timing: coincidence detection of oscillatory processes. Cogn. Brain Res. 21, 139-170. doi: 10.1016/j.cogbrainres.2004.06.012

Matthews, T. E., Witek, M. A. G., Heggli, O. A., Penhune, V. B., and Vuust, P. (2019). The sensation of groove is affected by the interaction of rhythmic and harmonic complexity. PLoS One 14:1. doi: 10.1101/415372

Matthews, T. E., Witek, M. A. G., Lund, T., Vuust, P., and Penhune, V. B. (2020). The sensation of groove engages motor and reward networks. NeuroImage 214:116768. doi: 10.1016/j.neuroimage.2020.116768

Merchant, H., Bartolo, R., Pérez, O., Méndez, J. C., Mendoza, G., Gámez, J., et al. (2014). "Neurophysiology of timing in the hundreds of milliseconds: multiple layers of neuronal clocks in the medial premotor areas," in Neurobiology of Interval Timing. Advances in Experimental Medicine and Biology, eds H. Merchant and V. de Lafuente (New York, NY: Springer), 143-154. doi: 10.1007/ 978-1-4939-1782-2_8

Merchant, H., Harrington, D. L., and Meck, W. H. (2013). Neural basis of the perception and estimation of time. Annu. Rev. Neurosci. 36, 313-336. doi: 10.1146/annurev-neuro-062012-170349

Merchant, H., and Honing, H. (2014). Are non-human primates capable of rhythmic entrainment? Evidence for the gradual audiomotor evolution hypothesis. Front. Neurosci. 7:274. doi: 10.3389/fnins.2013. 00274 
Meyer, L. B. (1956). Emotion and Meaning in Music. Chicago, IL: The University of Chicago Press.

Morillon, B., and Baillet, S. (2017). Motor origin of temporal predictions in auditory attention. Proc. Natl. Acad. Sci. U.S.A. 114, E8913-E8921. doi: 10.1073/ pnas. 1705373114

Nozaradan, S., Schwartze, M., Obermeier, C., and Kotz, S. A. (2017). Specific contributions of basal ganglia and cerebellum to the neural tracking of rhythm. Cortex 95, 156-168. doi: 10.1016/j.cortex.2017. 08.015

Pabst, A., and Balasubramaniam, R. (2018). Trajectory formation during sensorimotor synchronization and syncopation to auditory and visual metronomes. Exp. Brain Res. 236, 2847-2856. doi: 10.1007/s00221-018-5343-y

Parr, T., and Friston, K. J. (2019). Generalised free energy and active inference. Biol. Cybernet. 113, 495-513. doi: 10.1007/s00422-019-00805-w

Patel, A. D., and Iversen, J. R. (2014). The evolutionary neuroscience of musical beat perception: the Action Simulation for Auditory Prediction (ASAP) hypothesis. Front. Syst. Neurosci. 8:57. doi: 10.3389/fnsys.2014.00057

Perez, O., Kass, R., and Merchant, H. (2013). Trial time warping to discriminate stimulus-related from movement-related neural activity. J. Neurosci. Methods 212, 203-210. doi: 10.1016/j.jneumeth.2012.10.019

Polak, R., Jacoby, N., Fischinger, T., Goldberg, D., Holzapfel, A., and London, J. (2018). Rhythmic prototypes across cultures: a comparative study of tapping synchronization. Music Percept. 36, 1-23. doi: 10.1525/mp.2018.36.1.1

Povel, D. J., and Essens, P. (1985). Perception of temporal patterns. Music Percept. 2, 411-440. doi: $10.2307 / 40285311$

Rao, R. P. N., and Ballard, D. H. (1999). Predictive coding in the visual cortex: a functional interpretation of some extra-classical receptive-field effects. Nat. Neurosci. 2, 79-87. doi: 10.1038/4580

Rauschecker, J. P. (2011). An expanded role for the dorsal auditory pathway in sensorimotor control and integration. Hear. Res. 271, 16-25. doi: 10.1016/j. heares.2010.09.001

Rauschecker, J. P. (2018). Where, when, and how: are they all sensorimotor? Towards a unified view of the dorsal pathway in vision and audition. Cortex 98, 262-268. doi: 10.1016/j.cortex.2017.10.020

Rauschecker, J. P., and Tian, B. (2000). Mechanisms and streams for processing of "what" and "where" in auditory cortex. Proc. Natl. Acad. Sci. U.S.A. 97, 11800-11806. doi: 10.1073/pnas.97.22. 11800

Ravignani, A., Dalla-Bella, S., Falk, S., and Kello, C. (2019). Rhythm in speech and animal vocalizations: a cross-species perspective: speech rhythm across species. Ann. N. Y. Acad. Sci. 1452, 79-98. doi: 10.1111/nyas. 14166

Ross, J. M., Iversen, J., and Balasubramaniam, R. (2018a). Dorsal premotor contributions to auditory rhythm perception: causal transcranial magnetic stimulation studies of interval, tempo, and phase. bioRxiv [Preprint]. doi: 10. $1101 / 368597$

Ross, J. M., Iversen, J. R., and Balasubramaniam, R. (2018b). The role of posterior parietal cortex in beat-based timing perception: a continuous theta burst stimulation study. J. Cogn. Neurosci. 30, 634-643. doi: 10.1162/jocn_a_01237

Ross, J. M., Iversen, J. R., and Balasubramaniam, R. (2016a). Motor simulation theories of musical beat perception. Neurocase 22, 558-565. doi: 10.1080/ 13554794.2016.1242756
Ross, J. M., Warlaumont, A. S., Abney, D. H., Rigoli, L. M., and Balasubramaniam, R. (2016b). Influence of musical groove on postural sway. J. Exp. Psychol. Hum. Percept. Perform. 42, 308-319. doi: 10.1037/xhp0000198

Sarno, S., de Lafuente, V., Romo, R., and Parga, N. (2017). Dopamine reward prediction error signal codes the temporal evaluation of a perceptual decision report. Proc. Natl. Acad. Sci. U.S.A. 114, E10494-E10503. doi: 10.1073/pnas. 1712479114

Schubotz, R. I. (2007). Prediction of external events with our motor system: towards a new framework. Trends Cogn. Sci. 11, 211-218. doi: 10.1016/j.tics. 2007.02.006

Schultz, W. (2007). Multiple dopamine functions at different time courses. Ann. Rev. Neurosci. 30, 259-288. doi: 10.1146/annurev.neuro.28.061604.135722

Stupacher, J., Hove, M. J., Novembre, G., Schütz-Bosbach, S., and Keller, P. E. (2013). Musical groove modulates motor cortex excitability: a TMS investigation. Brain Cogn. 82, 127-136. doi: 10.1016/j.bandc.2013. 03.003

Sussman, E., Winkler, I., and Schröger, E. (2003). Top-down control over involuntary attention switching in the auditory modality. Psychon. Bull. Rev. 1, 630-637. doi: 10.3758/BF03196525

Tal, I., Large, E. W., Rabinovitch, E., Wei, Y., Schroeder, C. E., Poeppel, D., et al. (2017). Neural entrainment to the beat: the "missing-pulse" phenomenon. J. Neurosci. 37, 6331-6341. doi: 10.1523/JNEUROSCI.2500-16. 2017

Tanji, J. (2001). Sequential organization of multiple movements: involvement of cortical motor areas. Annu. Rev. Neurosci. 24, 631-651. doi: 10.1146/annurev. neuro.24.1.631

Teki, S., Grube, M., Kumar, S., and Griffiths, T. D. (2011). Distinct neural substrates of duration-based and beat-based auditory timing. J. Neurosci. 10, 3805-3812. doi: 10.1523/JNEUROSCI.5561-10.2011

Thompson, E. (2007). Mind in Life: Biology, Phenomenology, and the Sciences of Mind. Cambridge, MA: Harvard University Press.

van der Weij, B., Pearce, M. T., and Honing, H. (2017). A probabilistic model of meter perception: simulation enculturation. Front. Psychol. 8:824. doi: 10.3389/ fpsyg.2017.00824

Vuust, P., and Witek, M. A. G. (2014). Rhythmic complexity and predictive coding: a novel approach to modeling rhythm and meter perception in music. Front. Psychol. 5:1111. doi: 10.3389/fpsyg.2014.01111

Winkler, I., Háden, G. P., Ladinig, O., Sziller, I., and Honing, H. (2009). Newborn infants detect the beat in music. Proc. Natl. Acad. Sci. U.S.A. 106, 2468-2471. doi: 10.1073/pnas.0809035106

Conflict of Interest: The authors declare that the research was conducted in the absence of any commercial or financial relationships that could be construed as a potential conflict of interest.

Copyright () 2020 Proksch, Comstock, Médé, Pabst and Balasubramaniam. This is an open-access article distributed under the terms of the Creative Commons Attribution License (CC BY). The use, distribution or reproduction in other forums is permitted, provided the original author(s) and the copyright owner(s) are credited and that the original publication in this journal is cited, in accordance with accepted academic practice. No use, distribution or reproduction is permitted which does not comply with these terms. 